\title{
Study and analysis of Differential GNSS and Precise Point Positioning
}

\author{
Abhijit Dey ${ }^{1}$, Dr.V.Malleswara Rao ${ }^{2}$ \\ ${ }^{I}$ (Department of Electronics \& Communication, GITAM University, INDIA) \\ ${ }^{2}$ (Department of Electronics \& Communication, GITAM University, INDIA)
}

\begin{abstract}
The Global Positioning system (GPS) is used for navigation and associated tasks in wide variety of applications. One of the primary modes of GPS operation is differential GPS. Differential GPS allows more accurate positioning than stand-alone GPS. Many new applications are possible because of the increased accuracy obtained through differential GPS. In this paper the techniques and methods of differential GPS along with the algorithms are discussed which are providing more accurate and precise positioning in recent trends of communication. The purpose of the paper is to introduce the principles of differential GPS theory, and to provide a background for more advanced material.
\end{abstract}

Keywords: DGNSS (Differential navigation satellite system), PPP (Precise point positioning), RR (Reference receiver), UR (User receiver)

\section{INTRODUCTION}

The purpose of this work is to understand Differential GNSS algorithms, precise point positioning for better positioning and to obtain in real time, the position coordinates of the stationary "user" GPS receiver applying corrections given by the differential GPS technique (DGPS), as well as to compare different methods of DGPS implementations. Although the Global Positioning (GPS) was originally developed for navigation purposes, it has also shown to be an important source of information for vehicle attitude determination.

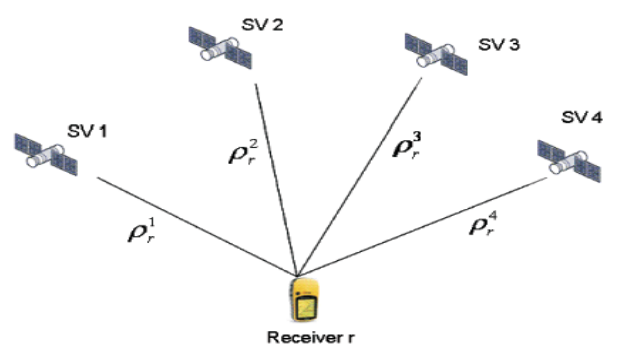

Fig 1: GPS satellites and receiver for coordinates.

Today, GPS aims to provide continuous 24 hours a day and 365 days a year 3-Dimensional positioning whether real-time or post processing at any location all over the world. Because the receiver clock is not synchronized with the satellite clock, this synchronization is the reason for the term "pseudo range" which measured via code and/or carrier phase. So, normally at least four satellites have to be observed at the same time to determine the 3D position and receiver clock bias, which is showed in Figure 1, where SV1, SV2, SV3, and SV4 are four satellites, $\rho_{\mathrm{r}}^{1}$ to $\rho_{\mathrm{r}}^{4}$ are pseudoranges from corresponding satellites to receiver $\mathrm{r}$.

GPS radio waves experience several changes as they pass through the ionosphere. A quiet ionosphere introduces the well-known code delay and phase advance effects.

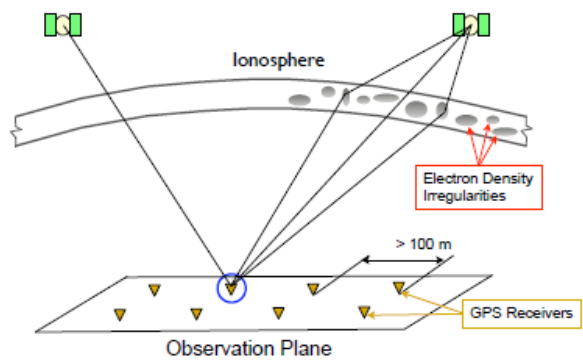

Fig 2: The undisturbed and disturbed ionosphere and the scintillation imaging array. 
The left half of Fig. 2 depicts the scenario where a GPS signal passes through a quiet portion of the ionosphere to a receiver situated on the ground.

The differential GPS technique consists of two major steps: 1) generating corrections on a reference "base"; 2) applying the corrections on the "user" receiver. The basic idea behind DGPS assumes that if two receivers are relatively close to each other (about up to $150 \mathrm{~km}$ ), the GPS radio signals will travel in very close paths, thus the atmospheric (ionosphere and troposphere) effects will be practically the same. These effects are spatially correlated, i. e., the magnitude error is dependent of the baseline length. The application of the corrections to both receivers makes therefore the final result more accurate (Strang and Borre, 1997).In this DGPS technique care of using only the simultaneously visible satellites for both receivers should be taken. DGPS techniques. This investigation paves the basis to develop a real time differential GPS system to be further implemented in dynamically mobile platforms, like aircraft, ships, and cars.

\section{DIFFERENTIAL GPS}

Differential GPS involves the use of two GPS receivers. One receiver is located at a fixed position that is accurately surveyed, while the other is allowed to move around. The goal is to accurately determine the position of the roaming receiver.

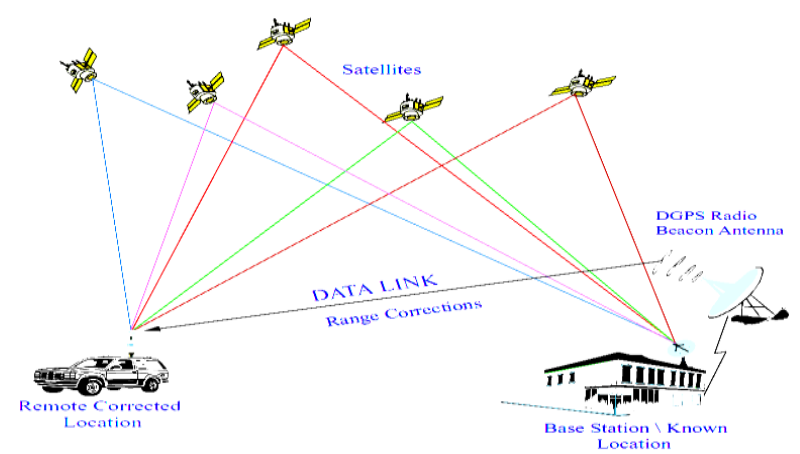

Fig 3: General representation of differential GPS

Differential GPS (DGPS) is a relatively simple technique to improve positional accuracy and integrity. This technique was developed in early 1980's and it is widely used in various forms. The system consists of a Reference Receiver (RR) located at a known location that has been previously surveyed, and one or more DGPS User Receivers (UR). The RR antenna, differential correction processing system, and datalink equipment (if used) are collectively called the Reference Station (RS). Both the UR and the RR data can be collected and stored for later processing, or sent to the desired location in real time via the data link. DGPS is based on the principle that receivers in the same vicinity will simultaneously experience common errors on a particular satellite ranging signal. In general, the UR (mobile receivers) use measurements from the RR to remove the common errors. In order to accomplish this, the UR must simultaneously use a subset or the same set of satellites as the reference station. The DGPS positioning equations are formulated so that the common errors cancel.

\subsection{Differencing Techniques [3]}

\subsubsection{Single differencing}

The purpose of single differencing is to eliminate satellite clock bias. Consider the observation equations for two receivers, A and B observing same satellite, $\mathrm{j}$ :

$\mathrm{L}_{\mathrm{A}}^{\mathrm{j}}=\rho_{\mathrm{A}}^{\mathrm{j}}+\mathrm{c} \tau_{\mathrm{A}}-\mathrm{c} \tau^{\mathrm{j}}+\mathrm{Z}_{\mathrm{A}}^{\mathrm{j}}-\mathrm{I}_{\mathrm{A}}^{\mathrm{j}}+\mathrm{B}_{\mathrm{A}}^{\mathrm{j}}$

$\mathrm{L}_{\mathrm{B}}^{\mathrm{j}}=\rho_{\mathrm{B}}^{\mathrm{j}}+\mathrm{c} \tau_{\mathrm{B}}-\mathrm{c} \tau^{\mathrm{j}}+\mathrm{Z}_{\mathrm{B}}^{\mathrm{j}}-\mathrm{I}_{\mathrm{B}}^{\mathrm{j}}+\mathrm{B}^{\mathrm{j}}{ }_{\mathrm{B}}$

The single difference phase is defined as the difference between these two:

$\Delta \mathrm{L}_{\mathrm{AB}=}^{\mathrm{j}}=\mathrm{L}_{\mathrm{A}-\mathrm{L}_{\mathrm{B}}^{\mathrm{j}}}^{\mathrm{j}}$

$=\left(\rho_{A}^{j}+c \tau_{A}-c \tau^{j}+Z_{A}^{j}-I_{A}^{j}+B_{A}^{j}-\left(\rho_{B}^{j}+c \tau_{B}-c \tau^{j}+Z_{B}^{j}{ }_{B} I_{B}^{j}+B_{B}^{j}\right)\right.$

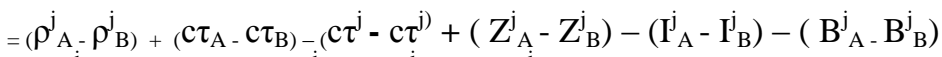

$=\Delta \rho_{\mathrm{AB}}^{\mathrm{j}}+\mathrm{c} \Delta \tau_{\mathrm{AB}}+\Delta \mathrm{Z}_{\mathrm{AB}}^{\mathrm{j}}-\Delta \mathrm{I}_{\mathrm{AB}}^{\mathrm{j}}+\Delta \mathrm{B}_{\mathrm{AB}}^{\mathrm{j}}$

where we use the double-subscript to denote quantities identified with two receivers, and the triangular symbol as a mnemonic device, to emphasise that the difference is made between two points on the ground. The geometry of single differencing is illustrated in Fig 4. 


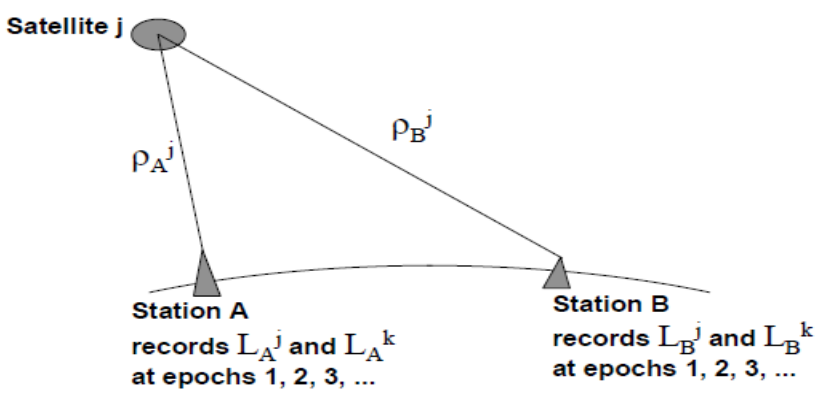

Fig 4: Single difference method.

\subsubsection{Double Differencing}

The purpose of "double differencing" is to eliminate receiver clock bias. Consider the single differenced observation equations for two receivers $A$ and $B$ observing satellites $j$ and $k$ :

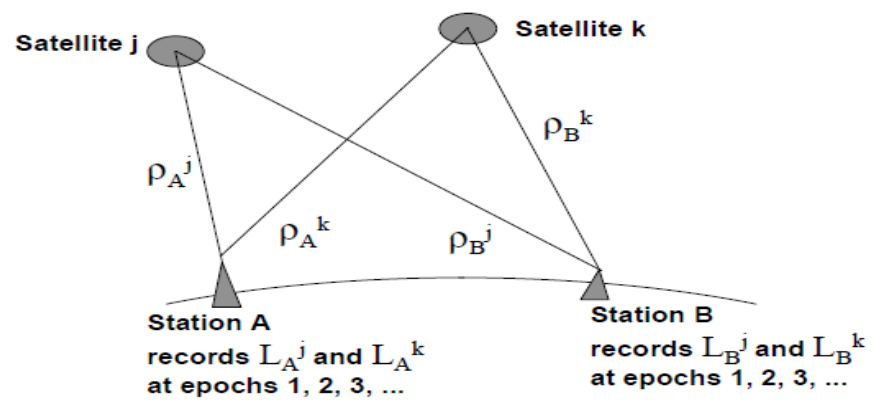

$\Delta \mathrm{L}^{\mathrm{j}}{ }_{\mathrm{AB}}=\Delta \rho^{\mathrm{j}}{ }_{\mathrm{AB}}+\mathrm{c} \Delta \tau_{\mathrm{AB}}+\Delta \mathrm{Z}^{\mathrm{j}} \mathrm{AB}-\Delta \mathrm{I}_{\mathrm{AB}}^{\mathrm{j}}+\Delta \mathrm{B}^{\mathrm{j}}{ }_{\mathrm{AB}}$

Fig 5: Double difference method.

$\Delta \mathrm{L}^{\mathrm{k}}{ }_{\mathrm{AB}}=\Delta \rho^{\mathrm{k}}{ }_{\mathrm{AB}}+\mathrm{c} \Delta \tau_{\mathrm{AB}}+\Delta \mathrm{Z}^{\mathrm{k}}{ }_{\mathrm{AB}}-\Delta \mathrm{I}^{\mathrm{k}}{ }_{\mathrm{AB}}+\Delta \mathrm{B}^{\mathrm{k}}{ }_{\mathrm{AB}}$

The double difference phase is defined as the difference between these two:

$$
\begin{aligned}
& \boldsymbol{\nabla} \Delta \mathrm{L}^{\mathrm{jk}}{ }_{\mathrm{AB}}=\Delta \mathrm{L}_{\mathrm{AB}}^{\mathrm{j}}-\Delta \mathrm{L}_{\mathrm{AB}}^{\mathrm{k}} \\
& =\left(\Delta \rho_{\mathrm{AB}}^{\mathrm{j}}+\mathrm{c} \Delta \tau_{\mathrm{AB}}+\Delta \mathrm{Z}_{\mathrm{AB}}^{\mathrm{j}}-\Delta \mathrm{I}_{\mathrm{AB}}^{\mathrm{j}}+\Delta \mathrm{B}^{\mathrm{j}}{ }_{\mathrm{AB}}\right) \\
& -\left(\Delta \rho^{\mathrm{k}}{ }_{\mathrm{AB}}+\mathrm{c} \Delta \tau_{\mathrm{AB}}+\Delta \mathrm{Z}^{\mathrm{k}}{ }_{\mathrm{AB}}-\Delta \mathrm{I}^{\mathrm{k}}{ }_{\mathrm{AB}}+\Delta \mathrm{B}^{\mathrm{k}}{ }_{\mathrm{AB}}\right) \\
& =\left(\Delta \rho^{\mathrm{j}}{ }_{\mathrm{AB}}-\Delta \rho^{\mathrm{k}}{ }_{\mathrm{AB}}\right)+\left(\mathrm{c} \Delta \tau_{\mathrm{AB}}-\mathrm{c} \Delta \tau_{\mathrm{AB}}\right)+\left(\Delta \mathrm{Z}_{\mathrm{AB}}^{\mathrm{j}}-\Delta \mathrm{Z}^{\mathrm{k}}{ }_{\mathrm{AB}}\right)-\left(\Delta \mathrm{I}_{\mathrm{AB}}^{\mathrm{j}}-\Delta \mathrm{I}_{\mathrm{AB}}^{\mathrm{k}}\right)-\left(\Delta \mathrm{B}_{\mathrm{AB}}^{\mathrm{j}}-\Delta \mathrm{B}^{\mathrm{k}}{ }_{\mathrm{AB}}\right) \\
& =\boldsymbol{\nabla} \Delta \mathrm{\rho}^{\mathrm{jk}}{ }_{\mathrm{AB}}+\boldsymbol{\nabla} \Delta \mathrm{Z}^{\mathrm{jk}}{ }_{\mathrm{AB}}-\boldsymbol{\nabla} \Delta \mathrm{i}_{\mathrm{AB}}^{\mathrm{jk}}+\boldsymbol{\nabla} \Delta \mathrm{B}^{\mathrm{jk}}{ }_{\mathrm{AB}}
\end{aligned}
$$

where we use the double-superscript to denote quantities identified with two satellites, and the upside-down triangular symbol as a mnemonic device, to emphasise that the difference is made between two points in the sky. Fig 5 illustrates the geometry of double differencing.

\subsubsection{Double Differenced Ambiguity}

The double difference combination has an additional advantage, in that the ambiguity is an integer:

$$
\begin{aligned}
& \boldsymbol{\nabla} \Delta \mathrm{B}^{\mathrm{jk}}{ }_{\mathrm{AB}}=\Delta \mathrm{B}^{\mathrm{j}}{ }_{\mathrm{AB}}-\Delta \mathrm{B}^{\mathrm{k}}{ }_{\mathrm{AB}} \\
& =\left(B_{A}^{j}-B_{B}^{j}\right)-\left(B^{k}{ }_{A}-B_{B}^{k}\right) \\
& =\lambda_{0}\left(\varphi_{0 A}-\varphi_{o}^{j}-N_{A}^{j}\right)-\lambda_{0}\left(\varphi_{o B}-\varphi_{o}^{j}-N_{B}^{j}\right)-\lambda_{0}\left(\varphi_{o A}-\varphi_{o}^{k}-N_{A}^{k}\right)+\lambda_{o}\left(\varphi_{o B}-\varphi_{0}^{k}-N_{B}^{k}\right) \\
& =-\lambda_{\mathrm{o}}\left(\mathrm{N}_{\mathrm{A}}^{\mathrm{j}}-\mathrm{N}_{\mathrm{B}}^{\mathrm{j}}-\mathrm{N}_{\mathrm{A}}^{\mathrm{k}}-\mathrm{N}_{\mathrm{B}}^{\mathrm{k}}\right) \\
& =-\lambda_{\mathrm{o}} \boldsymbol{\nabla} \Delta \mathrm{N}_{\mathrm{AB}}^{\mathrm{jk}}
\end{aligned}
$$

Hence we can write the double differenced phase observation equation :

$\nabla \Delta \mathrm{L}^{\mathrm{jk}}{ }_{\mathrm{AB}}=\boldsymbol{\nabla} \Delta \rho^{\mathrm{jk}}{ }_{\mathrm{AB}}+\mathbf{\nabla} \Delta \mathrm{Z}^{\mathrm{jk}}{ }_{\mathrm{AB}}-\mathbf{\nabla} \Delta \mathrm{I}^{\mathrm{jk}}{ }_{\mathrm{AB}}-\lambda_{\mathrm{o}} \nabla \Delta \mathrm{N}^{\mathrm{jk}}{ }_{\mathrm{AB}}$

From the point of view of estimation, it makes no difference whether we use a minus or plus sign for $\mathrm{N}$, so long as the partial derivative has a consistent sign (which, for the above equation, would be $-\lambda \mathrm{o}$ ). 


\subsubsection{Triple Differencing}

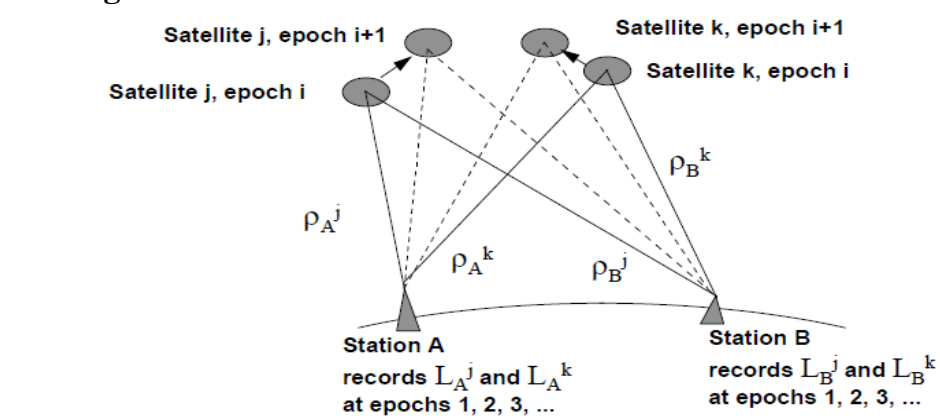

Fig 6: Triple difference method.

$$
\begin{aligned}
\boldsymbol{\nabla} \Delta \mathrm{L}^{\mathrm{jk}}{ }_{\mathrm{AB}}(i) & =\boldsymbol{\nabla} \Delta \rho^{\mathrm{jk}}{ }_{\mathrm{AB}}(i)+\boldsymbol{\nabla} \Delta \mathrm{Z}^{\mathrm{jk}}{ }_{\mathrm{AB}}(i)-\boldsymbol{\nabla} \Delta \mathrm{I}^{\mathrm{jk}}{ }_{\mathrm{AB}}(i)-\lambda_{\mathrm{o}} \boldsymbol{\nabla} \Delta \mathrm{N}^{\mathrm{jk}}{ }_{\mathrm{AB}} \\
\boldsymbol{\nabla} \Delta \mathrm{L}^{\mathrm{jk}}{ }_{\mathrm{AB}}(i+1) & =\boldsymbol{\nabla} \Delta \rho^{\mathrm{jk}}{ }_{\mathrm{AB}}(i+1)+\boldsymbol{\nabla} \Delta \mathrm{Z}^{\mathrm{jk}}{ }_{\mathrm{AB}}(i+1)-\boldsymbol{\nabla} \Delta \mathrm{I}^{\mathrm{k}}{ }_{\mathrm{AB}}(i+1)-\lambda_{\mathrm{o}}{ }^{\mathrm{jk}} \Delta \mathrm{N}{ }_{\mathrm{AB}}
\end{aligned}
$$

The triple difference phase is defined as the difference between these two:

$\delta(i, i+1) \nabla \Delta \mathrm{L}^{\mathrm{jk}}{ }_{\mathrm{AB}}=\boldsymbol{\nabla} \Delta \mathrm{L}^{\mathrm{jk}}{ }_{\mathrm{AB}}(i+1)-\boldsymbol{\nabla} \Delta \mathrm{L}^{\mathrm{jk}}{ }_{\mathrm{AB}}(i)$

$$
=\delta(i, i+1) \boldsymbol{\nabla} \Delta \rho^{\mathrm{jk}}{ }_{\mathrm{AB}}(i)+\delta(i, i+1) \boldsymbol{\nabla} \Delta \mathrm{Z}^{\mathrm{jk}}{ }_{\mathrm{AB}}(i)-\delta(i, i+1) \boldsymbol{\nabla} \Delta \mathrm{I}^{\mathrm{jk}}{ }_{\mathrm{AB}}(i)
$$

where we use the delta symbol to indicate the operator that differences data between epochs. Fig 6 illustrates triple differencing geometry.

All DGNSS techniques perform positioning relative to one or more reference GNSS receivers located at points of known coordinates. Depending upon the type of GNSS measurement, user equipment, reference receiver infrastructure, data processing algorithm, ancillary products or services, and operational requirements (e.g. realtime or post mission, moving or static user equipment, good or poor satellite and reference receiver geometry, etc.), different levels of performance are obtained. For example, the basic DGNSS technique using singlefrequency pseudo-range measurements can deliver few-metre to submetre positioning accuracy, in either localarea DGNSS or wide-area DGNSS implementations.

The processing of carrier phase data enables sub-decimeter-level accuracy, with the highest operational accuracy being possible (at the few-centimeter level) using the most sophisticated receiver equipment and algorithms, even in real-time and with the user equipment in motion (the so-called RTK mode).

Over the time period the technique of Precise Point Positioning (PPP) has been touted as an alternative for the "infrastructure-hungry" carrier phase-based DGNSS techniques. Although PPP suffers from several disadvantages - slow convergence times, no user equipment supports real-time algorithms, no real-time satellite orbit and clock data streams, uncertain coordinate datum - recent PPP developments could address most of these disadvantages.

For computing range and range rate corrections at a base station. Let $\rho$ denote the geometric distance between the satellite and the receiver antennas, $\mathrm{dt}_{\mathrm{i}}$ denote the receiver clock offset $\mathrm{dt}^{\mathrm{k}}$ the satellite clock offset, and $\mathrm{T}$ the tropospheric delay. Then the corrected range is computed as [4]

$$
\rho^{*}=\rho+\mathrm{cdt}_{\mathrm{i}}-\mathrm{cdt}^{\mathrm{k}}+\mathrm{T}
$$

and the range correction as

$$
d=\rho *-P_{o b s}
$$

The fig 7 shows the range and range corrections over 200 epochs. The plot is obtained by using Matlab R2012b. It shows the differential corrections of each satellite at the base station.IIsc Bangalore January 1, 2014 data is used for the analysis. 

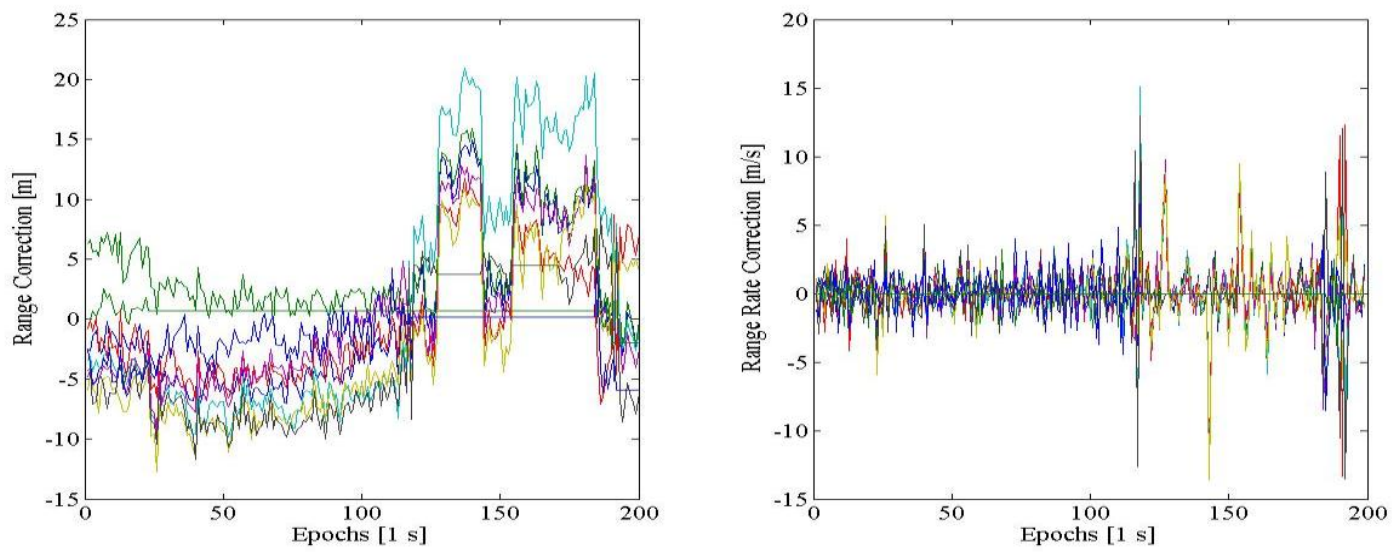

Fig 7: shows range and range rate corrections over 200 epochs using January 1, 2014, data [IISC Bangalore]

\section{Precise Point Positioning}

The Precise point positioning (PPP) is a method that performs precise position determination using a single GPS receiver. This positioning approach arose from the advent of widely available precise GPS orbit and clock data products with centimeter accuracy. These data can be applied to substantially reduce the errors in GPS satellite orbits and clocks, two of the most significant error sources in GPS positioning.

Combining precise satellite positions and clocks with a dual-frequency GPS receiver (to remove the first order effect of the ionosphere), PPP is able to provide position solutions at centimeter to decimeter level, which is appealing to many applications such as airborne mapping. PPP is different from double-difference RTK (realtime kinematic) positioning that requires access to observations from one or more base stations with known coordinates. The word "precise" is also used to distinguish it from the conventional point positioning techniques that use only code or phase smoothed code as the principal observable for position determination.

The PPP method can offer several significant advantages to applications compared to differential precise positioning techniques. First, PPP involves only a single GPS receiver and, therefore, removes the need for GPS users to establish local base stations. As a result, it eliminates the spatial operating range limit as well as the constraint of simultaneous observations on both rover and base receivers imposed by the differential RTK technique.

\subsection{Single-Frequency PPP}

Although the prospect of triple-frequency PPP is tantalising, such a capability is many years away from being a reality. On the other hand, dual frequency PPP has already demonstrated the capability of providing accurate position solutions at the sub-decimeter level for kinematic positioning and sub-centimeter level for static positioning. However, since the majority of the mass-market GNSS users (navigation and GIS) operate single-frequency GPS receivers, PPP using a single-frequency GPS receiver has the potential to open up PPP to a broader range of applications. The large errors in single-frequency PPP, after the application of precise GPS orbit and clock products, are mainly due to ionospheric effects which cannot be mitigated effectively using single-frequency measurements (Chen and Gao, 2005).Using the Klobuchar model, based on the ionospheric coefficients broadcast from the GPS satellites, is the simplest way to mitigate the ionospheric delay on measurements in single frequency PPP. However, only about 50-60\% of the total ionospheric effects are mitigated (Klobuchar, 1996).

IGS has generated products with information on Total Electron IGS has generated products with information on the Total Electron Content (TEC) of the ionosphere on a global scale since 1998 (http://igs.org/projects/iono/index.html).

\subsection{Dual-Frequency PPP}

Unlike with differential processing, in PPP the full effect of tropospheric delays must be estimated explicitly, resulting in an additional unknown zenith tropospheric delay (ZTD) parameter that must be estimated along with the position. Further, the standard approach in PPP for handling ionospheric delays is to use a dual-frequency receiver and form the "ionosphere-free" linear combination of L1 and L2 carrier phase and pseudorange observations. The PPP algorithm may use both the ionosphere-free linear combinations of carrier phase and pseudorange observations, or only the ionosphere-free linear combination of carrier phase observations. Using the pseudorange observations makes a difference for the initial convergence, but the final solution is almost fully dependent on the carrier phase data. 
The ionosphere-free linear combination is very noisy as it amplifies multipath and receiver measurement errors. The ionosphere-free linear combination is also very costly in terms of redundancy: from four dual-frequency code and carrier phase observations, only two ionosphere-free observations remain. In fact, if both the ionosphere-free linear combination of code and carrier phase data are formed, the ionosphere delay is eliminated twice (in other words, we estimate the ionosphere delay once from the code measurements and once from the carrier phase measurements). Typically, the noise in the ionosphere-free linear combinations would be a factorthree higher compared to the original observations.

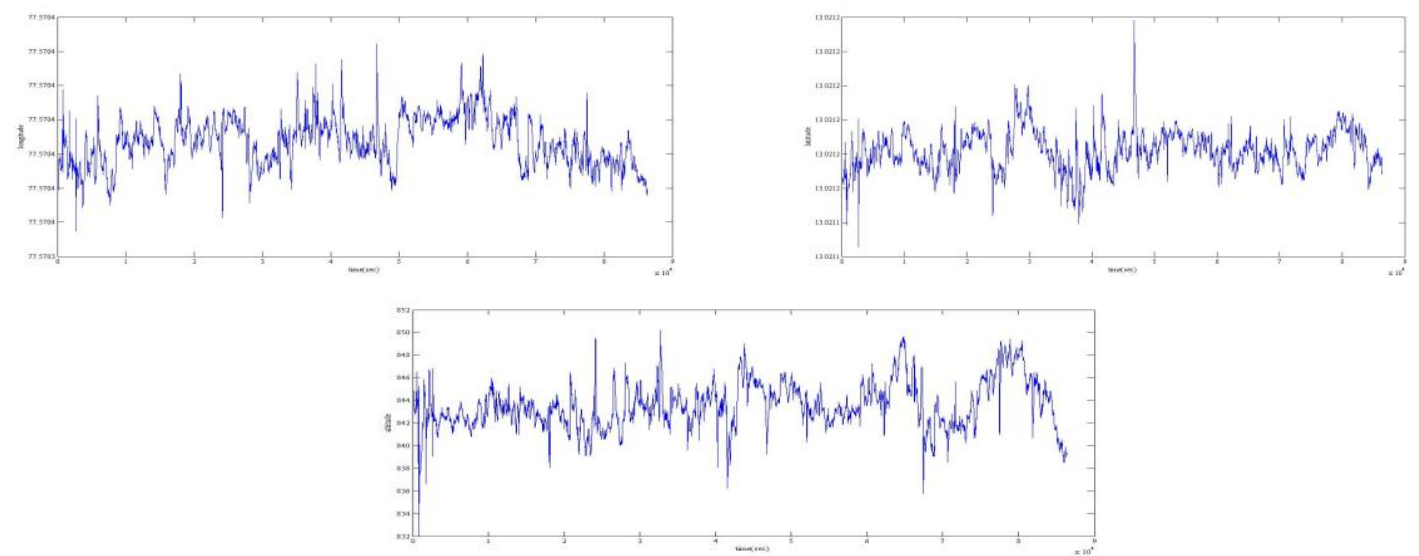

Fig 8: Using dual frequency L1 \& P1 precise point positioning (Lat long alt) using January 13, 2014 data [IISC

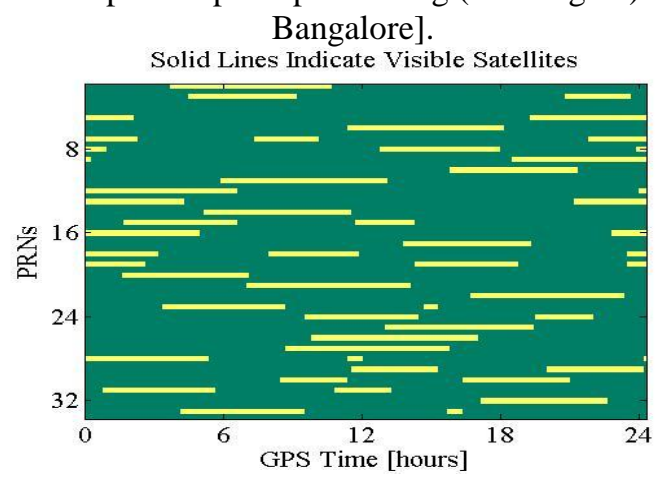

Fig 9: Number of visible satellites.

\section{RESULT AND DISCUSSIONS}

The concept of Differential GPS (DGPS) is understood along with DGPS algorithms which include single, double and triple difference method. The range and range rate corrections are done in Matlab environment. The output of the range rate corrections are shown in figure7.IIsc Bangalore January 1, 2014 Rinex (navigation and observation) data is used for the analysis. Satellite wise range correction is done, for this pseudo code $\mathrm{C} 1$ is used. A differential correction at the base station is computed as shown in figure 7.

Further the approach of precise point positioning is explained and using dual frequency (L1 \& P1) codes the PPP (Precise Point Positioning) is done in Matlab environment. The precise point is computed at every 30 seconds and figures 8 shows the computed PPP w.r.t time (seconds). In figure 8 the plots shows the latitude, longitude and altitude at every 30 seconds. Fig 9 shows the number of satellites available at that time. The solid lines indicate the visible satellites plotted w.r.t. GPS time in hours.

\section{Acknowledgements}

The authors of this paper would like to acknowledge all the corresponding IEEE paper holders and most importantly the publishers of related books and journals which gave immense support and inspiration in preparing this manuscript. Above all, the extreme mental support and source of inspiration from all the family members and friends are widely acknowledged.

\section{REFERENCES}

[1] Farrell, J.A., and M.Barth, The Global Positioning System and Inertial Navigation, McGraw-Hill, ISBN-0-07-022045-X, 1999.

[2] Kaplan, E., ed., Understanding GPS: Principles and Application Boston: Artech House, c1996

[3] Geoffrey Blewitt., Geodetic applications of GPS: Basics of GPS techniques, Swedish Land Survey

[4] Kai Borre, Easy GPS suite II:Inside GNSS,June 2010 
[5] Chao, C.H. (1998). "High Precision Differential GPS”. MSc Dissertation. Institute of Engineering Surveying and Space Geodesy (Institute of Engineering Surveying and Space Geodesy (IESSG)) -University of Nottingham.

[6] RTCM Special Committee No. 104. (1990). “RTCM Recommended Standards for Differential NAVSTAR GPS Service”. Radio Technical Committee for Maritime Services. Paper 134-89/SC104- 68. Washington DC (USA).

[7] Walsh, D. (1994). "Kinematic GPS Ambiguity Resolution”. PhD Thesis, Institute of Engineering Surveying and Space Geodesy (IESSG), University of Nottingham..

[8] Ko, P.Y. (1997). "Wide Area Differential GPS (WADGPS)". PhD Thesis. Stanford University (USA). 